Article

\title{
Mass in de Sitter and Anti-de Sitter Universes with Regard to Dark Matter
}

\author{
Jean-Pierre Gazeau
}

CNRS, Astroparticule et Cosmologie, Université de Paris, F-75013 Paris, France; gazeau@apc.in2p3.fr

Received: 6 April 2020; Accepted: 2 May 2020; Published: 5 May 2020

\begin{abstract}
An explanation of the origin of dark matter is suggested in this work. The argument is based on symmetry considerations about the concept of mass. In Wigner's view, the rest mass and the spin of a free elementary particle in flat space-time are the two invariants that characterize the associated unitary irreducible representation of the Poincaré group. The Poincaré group has two and only two deformations with maximal symmetry. They describe respectively the de Sitter (dS) and anti-de Sitter (AdS) kinematic symmetries. Analogously to their shared flat space-time limit, two invariants, spin and energy scale for de Sitter and rest energy for anti-de Sitter, characterize the unitary irreducible representation associated with $\mathrm{dS}$ and AdS elementary systems, respectively. While the dS energy scale is a simple deformation of the Poincaré rest energy and so has a purely mass nature, AdS rest energy is the sum of a purely mass component and a kind of zero-point energy derived from the curvature. An analysis based on recent estimates on the chemical freeze-out temperature marking in Early Universe the phase transition quark-gluon plasma epoch to the hadron epoch supports the guess that dark matter energy might originate from an effective AdS curvature energy.
\end{abstract}

Keywords: de Sitter; anti-de Sitter; group representation; hadronization; dark matter; zero-point energy; quark-gluon plasma; critical point

MSC: 81R05; 85A04; 81V05

\section{Introduction: Some (Observational) Facts about Dark Matter}

According to the Planck 2018 analysis of CMB power spectrum [1], our Universe is spatially flat, accelerating, and composed of $5 \%$ baryonic matter, $27 \%$ cold dark matter (CDM, non baryonic), and $68 \%$ dark energy $(\Lambda)$. (Cold) dark matter [2] is observed by its gravitational influence on luminous, baryonic matter. The dark matter mass halo and the total stellar mass are coupled through a function that varies smoothly with mass (see [3] and references therein), with possible noticeable exception(s) like the recent [4-6]. Up to now, all hypothetical particle models (WIMP, Axions, Neutrinos ...) failed direct or indirect detection tests. Alternative theories (e.g., MOND), which negate the existence of dark matter as a physical entity, have failed to explain clusters and the observed pattern in the CMB.

In this article, we view dark matter as a physical entity and we propose an explanation of its current existence as the remnant, after hadronization, of the zero-point energy of the quark-gluon plasma (QGP) [7] due to an effective anti-de-Sitterian environment experienced by the QGP massive constituents existing at the so-called quark epoch, over about $10^{-12} \mathrm{~s}$ till $10^{-6} \mathrm{~s}$ after the Big-Bang and subsequent to the inflation (over about $10^{-33} \mathrm{~s}$ till $10^{-32} \mathrm{~s}$ ).

Section 2 is a survey of Poincaré, de Sitter, and anti-de Sitter kinematic symmetries and their quantum realizations in terms of the unitary irreducible representations (UIR) of the corresponding relativity groups which are labeled by spin and $\sim$ mass invariants. In Section 3, these de Sitter and anti-de Sitter massive representations are considered from the point of view of their Minkowskian contraction limits in terms of the so-called Garidi mass formulas. We then reexamine in Section 4 the 
dark matter enigma by advancing three hypotheses based on the anti-de Sitter Garidi mass formula and the experimental evidence of the phase transition from hadronic confinement to QGP deconfinement. We conclude in Section 5 with a few comments.

\section{Mass and Symmetries}

In Minkowski (M), the concept of (rest) mass originates from the ubiquitous law of conservation of energy, a direct consequence of the Poincaré symmetry. This concept was formulated by Wigner [8] in rigorous mathematical terms as associated with the concept of elementary system. The latter is a description of a set of states which forms a unitary irreducible representation (UIR) space for the proper orthochronous Poincaré group $\mathrm{P}_{0}^{\uparrow}=\mathbb{R}^{1,3} \rtimes \mathrm{SO}_{0}(1,3)$ (or $\mathbb{R}^{1,3} \rtimes \mathrm{SL}(2, \mathbb{C})$ ), semi-direct product of the translations in Minkowski with the Lorentz group (or its universal covering). The UIRs of the Poincaré group [9] that we are concerned with here are the massive ones with positive energy. They are denoted by $U_{\mathrm{M}}(m, s)$ and are completely characterized by the eigenvalues of two Casimir operators, namely the quadratic Klein-Gordon operator

$$
Q_{\mathrm{M}}^{(1)}=P^{\mu} P_{\mu}=P_{0}^{2}-\mathbf{P}^{2},
$$

with eigenvalues $\left\langle Q_{\mathrm{M}}^{(1)}\right\rangle=m^{2} c^{2}$, and the quartic Pauli-Lubanski operator

$$
Q_{\mathrm{M}}^{(2)}=W^{\mu} W_{\mu}, W_{\mu}=\frac{1}{2} \epsilon_{\mu \nu \rho \sigma} J^{v \rho} P^{\sigma}
$$

with eigenvalues (in the non-zero mass case) $\left\langle Q_{M}^{(2)}\right\rangle=-m^{2} c^{2} s(s+1) \hbar^{2}$.

As it was explained in [10], with the requirements of kinematical rotation, parity, and time-reversal invariance, there exists one way only to deform the Poincaré group $\mathrm{P}_{0}^{\uparrow}$, namely, in endowing space-time with a certain curvature. Hence, there are two possible deformations that are distinguished with the sign of curvature of the corresponding space-times, namely the de Sitter (dS) and anti-de Sitter (AdS) space-times [11,12]. More precisely, dS (resp. AdS) are the unique maximally symmetric solutions of the vacuum Einstein's equations with positive (resp. negative) cosmological constant $\Lambda$ [13]. Their symmetries are one-parameter deformations of the Minkowskian symmetry with

- negative curvature $-\varkappa_{\mathrm{dS}}=-H / c=-\sqrt{\Lambda_{\mathrm{dS}} / 3}$

- $\quad$ positive curvature $\varkappa_{\text {AdS }}=\sqrt{\left|\Lambda_{\text {AdS }}\right| / 3}$

Their respective invariances (in the relativity or kinematical sense) hold with respect to the ten-parameter $\mathrm{dS}$ group $\mathrm{SO}_{0}(1,4)$ (or its universal covering $\mathrm{Sp}(2,2)$ ) and the ten-parameter AdS group $\mathrm{SO}_{0}(2,3)$ (or its two-fold covering $\mathrm{Sp}(4, \mathbb{R})$, or even its universal covering) groups.

The "massive" UIR's of the dS [14,15] and AdS [16] groups are those which are deformations of the above $U_{\mathrm{M}}(m, s)$. Those UIRs are here denoted by $U_{\mathrm{dS}}\left(\varsigma_{\mathrm{dS}}, s\right)$ and $U_{\mathrm{AdS}}\left(\varsigma_{\mathrm{AdS}}, s\right)$, respectively. The real dimensionless parameters $\zeta_{\mathrm{dS}}$ and $\zeta_{\mathrm{AdS}}$ replace the Minkowskian rest mass. Together with the spin $s$, they determine the corresponding UIR through the eigenvalues of dS and AdS invariant Casimir operators [17]. The dS UIR $U_{\mathrm{dS}}\left(\varsigma_{\mathrm{dS}}, s\right)$ belongs to the so-called principal series, for which we have

$$
\left\langle Q_{\mathrm{dS}}^{(1)}\right\rangle=s_{\mathrm{dS}}^{2}+\frac{9}{4}-s(s+1), \quad\left\langle Q_{\mathrm{dS}}^{(2)}\right\rangle=\left(s_{\mathrm{dS}}^{2}+\frac{1}{4}\right) s(s+1)
$$

with $\varsigma_{\mathrm{d} S} \in \mathbb{R}$, and $s \in \mathbb{N} / 2$. Note that $U_{\mathrm{dS}}\left(\varsigma_{\mathrm{dS}}, 0\right)$ and $U_{\mathrm{dS}}\left(-\varsigma_{\mathrm{dS}}, 0\right)$ are equivalent. The AdS UIR $U_{\mathrm{AdS}}\left(\varsigma_{\mathrm{AdS}}, s\right)$ belongs to the so-called discrete series, and we have

$$
\left\langle Q_{\mathrm{AdS}}^{(1)}\right\rangle=\varsigma_{\mathrm{AdS}}\left(\varsigma_{\mathrm{AdS}}-3\right)+s(s+1), \quad\left\langle Q_{\mathrm{AdS}}^{(2)}\right\rangle=-\left(\varsigma_{\mathrm{AdS}}-1\right)\left(\varsigma_{\mathrm{AdS}}-2\right) s(s+1),
$$

with $s \in \mathbb{N} / 2$ and $\varsigma_{\text {AdS }}=s+2, s+3, \ldots$ (or $\varsigma_{\text {AdS }}>s+1$ for the universal covering of $S_{0}(2,3)$ ). 
There exists an irreconcilable difference between the dS invariant $\zeta_{\mathrm{dS}}$ and the AdS one $\varsigma_{\text {Ads }}$. The latter is the lowest value of the discrete spectrum of the generator of "time" rotations in AdS [18]. Hence, it can be given a non ambiguous meaning of a rest energy when it is expressed in energy AdS units $\hbar \varkappa_{\text {AdS }} / c$ :

$$
E_{\text {AdS }}^{\text {rest }}:=\hbar c \varkappa_{\text {AdS }} \zeta_{\text {AdS }} .
$$

Therefore, this physical concept of "energy at rest" survives with the deformation Poincaré $\rightarrow$ AdS.

The situation is radically different with dS. The spectrum of the generator of "time" hyperbolic rotations in $\mathrm{dS}$ is not bounded below [19]. Actually, it covers the whole real line. Of course, the invariant $\varsigma_{\mathrm{dS}}$ can be given an energy dimension as

$$
E_{\mathrm{dS}}:=\hbar c \varkappa_{\mathrm{dS}} \varsigma_{\mathrm{dS}} .
$$

\section{Minkowskian Content of dS and AdS Elementary Systems: The Garidi Mass}

If we wish to go further into the interpretative problem of a mass in a dS/AdS background, the crucial question to be addressed concerns the interpretation of the dS/AdS UIR's (or quantum AdS and dS elementary systems) from a (asymptotically) Minkowskian point of view. We mean by this the study of the contraction limit $\varkappa \rightarrow 0$ or equivalently $\Lambda \rightarrow 0$ of these representations. The notion of mass in "de Sitterian Physics" may appear ambiguous in terms of contraction of representation [20,21], exemplified by the fact that one cannot give a precise meaning to a dS rest energy, except if one follows an approach based on a causality de Sitterian semi-group [19], or based on a analyticity prerequisite [22]. Nevertheless, a consistent mass formula has been proposed by Garidi [23] in terms of the dS UIR parameters $\zeta_{\mathrm{dS}}$ and $s$ :

$$
m_{\mathrm{dS}}^{2}=\frac{\hbar^{2} \varkappa_{\mathrm{dS}}^{2}}{c^{2}}\left(\left\langle Q_{\mathrm{dS}}^{(1)}\right\rangle-\left\langle\left. Q_{\mathrm{dS}}^{(1)}\right|_{s=1 / 2+\mathrm{is}_{\mathrm{dS}}}\right\rangle\right)=\frac{\hbar^{2} \varkappa_{\mathrm{dS}}^{2}}{c^{2}}\left(\varsigma_{\mathrm{dS}}^{2}+\left(s-\frac{1}{2}\right)^{2}\right) .
$$

The minimal value assumed by the eigenvalues of the first Casimir in the set of UIR in the discrete series is precisely reached at $s=1 / 2+\mathrm{i} \varsigma_{\mathrm{dS}}$, which corresponds to the "conformal" massless case, for which $s$ clearly loses its spin meaning. Controlling the validity of such a formula from a Minkowskian observer amounts to understanding the contraction (mathematically non-trivial in terms of sequences of Hilbert spaces [24])

$$
\text { dS UIR } \longrightarrow \text { Poincaré UIR }
$$

Then, the contraction dS $\rightarrow$ Poincaré in terms of mass has to be understood as

$$
\varkappa_{\mathrm{dS}} \rightarrow 0 \text { and } \varsigma_{\mathrm{dS}} \rightarrow+\infty \text { while } \varsigma_{\mathrm{dS}} \hbar \varkappa_{\mathrm{dS}} / c \rightarrow m
$$

In terms of representations,

$$
D\left(\varsigma_{\mathrm{dS}}, s\right) \underset{\varkappa_{\mathrm{dS}} \rightarrow 0, \varsigma_{\mathrm{dS}} \rightarrow \infty}{\longrightarrow} U_{\mathrm{M}}(m, s) .
$$

Thus, close by the contraction limit,

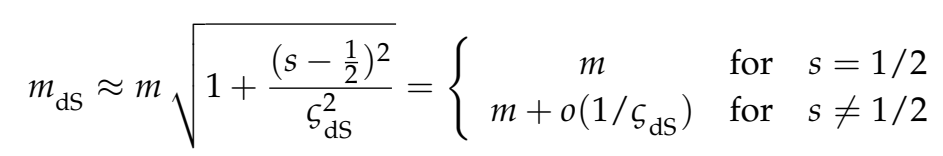


The analogous of the Garidi mass for the AdS case exists as well $[25,26]$. It precisely vanishes for massless conformal AdS fields that lie at the lowest limit $\varsigma$ AdS $=s+1$ of the discrete series.

$$
\begin{aligned}
m_{\mathrm{AdS}}^{2} & =\frac{\hbar^{2} \varkappa_{\mathrm{AdS}}^{2}}{c^{2}}\left(\left\langle Q_{\mathrm{AdS}}^{(1)}\right\rangle-\left\langle\left. Q_{\mathrm{AdS}}^{(1)}\right|_{\varsigma_{\mathrm{AdS}}=s+1}\right\rangle\right) \\
& =\frac{\hbar^{2} \varkappa_{\mathrm{AdS}}^{2}}{c^{2}}\left[\left(\varsigma_{\mathrm{AdS}}-\frac{3}{2}\right)^{2}-\left(s-\frac{1}{2}\right)^{2}\right] .
\end{aligned}
$$

As for $\mathrm{dS}$, the contraction AdS $\rightarrow$ Poincaré in terms of mass reads as

$$
\varkappa_{\text {AdS }} \rightarrow 0 \text { and } \varsigma_{\text {AdS }} \rightarrow+\infty \quad \text { while } \quad \varsigma_{\text {AdS }} \hbar \varkappa_{\text {AdS }} / c \rightarrow m
$$

The contraction AdS $\rightarrow$ Poincaré in terms of masses and representations reads as

$$
D\left(\varsigma_{\text {AdS }}, s\right) \underset{\substack{\varkappa_{\text {AdS }} \rightarrow 0, \varsigma_{\text {AdS }} \rightarrow \infty \\ \varsigma_{\text {AdS }} \hbar \varkappa_{\text {AdS }} / c \rightarrow m}}{\longrightarrow} U \text { M }(m, s) .
$$

We now show how the rest energy introduced in Equation (1) for a massive AdS elementary system reveals a universal pure curvature or vibration energy component besides a matter energy content. From the Garidi mass formula in Equation (4), an AdS elementary system can indeed be viewed asymptotically [27] as a combination of both a relativistic free particle with rest energy $m_{\text {Ads }} c^{2} \approx m c^{2}$ and a $3 \mathrm{D}$ isotropic quantum harmonic oscillator with zero-point energy $\frac{3}{2} \hbar \varkappa_{\text {AdS } C} \equiv \frac{3}{2} \hbar \omega_{\text {AdS }}$ at the first order in the curvature:

$$
\begin{aligned}
E_{\mathrm{AdS}}^{\mathrm{rest}} & =\hbar \varkappa_{\mathrm{AdS}} c S_{\mathrm{AdS}}=\left[m_{\mathrm{AdS}}^{2} c^{4}+\hbar^{2} \omega_{\mathrm{AdS}}^{2}\left(s-\frac{1}{2}\right)^{2}\right]^{1 / 2}+\frac{3}{2} \hbar \omega_{\mathrm{AdS}} \\
& =m_{\mathrm{AdS}} c^{2}+\frac{3}{2} \hbar \omega_{\mathrm{AdS}}+\frac{1}{2} \frac{\hbar^{2} \omega_{\mathrm{AdS}}^{2}}{m_{\mathrm{AdS}} c^{2}}\left(s-\frac{1}{2}\right)^{2}+o\left(\varkappa_{\mathrm{AdS}}^{2}\right) \\
& =m_{\mathrm{AdS}} c^{2}+\frac{3}{2} \hbar \omega_{\mathrm{AdS}}+o\left(\varkappa_{\mathrm{AdS}}\right) .
\end{aligned}
$$

By contrast, the meaning of energy in dS relativity with regard to its Poincare limit is less tractable. It is also exemplified by the absence of any term of order $\varkappa_{\text {AdS }}$ besides the $\mathrm{dS}$ mass energy

$$
E_{\mathrm{dS}}=\hbar \varkappa_{\mathrm{dS}} c S_{\mathrm{dS}}=m_{\mathrm{dS}} c^{2}+o\left(\varkappa_{\mathrm{dS}}\right)
$$

One notices the remarkable position occupied by the spin $s=1 / 2$ in the above formulas:

$$
\begin{array}{cl}
\text { for dS: } & E_{\mathrm{dS}}=m_{\mathrm{dS}} c^{2}, \\
\text { for AdS: } & E_{\mathrm{AdS}}^{\mathrm{rest}}=m_{\mathrm{AdS}} c^{2}+\frac{3}{2} \hbar \varkappa_{\mathrm{AdS}} c .
\end{array}
$$

\section{Dark Matter as a Relic AdS Curvature Energy?}

Dark matter is observed as an energy more or less localized in halos surrounding baryonic matter in galaxies and galaxy clusters.

Assumption 1. In our approach, the nature of this dark matter energy is supposed to be related to some effective AdS curvature. Precisely, for a spin 1/2 elementary particle $X$,

$$
E_{\text {AdS }}^{\text {rest }}=\underbrace{m_{\text {AdS }}(X) c^{2}}_{\text {visible }}+\underbrace{\frac{3}{2} \hbar \varkappa_{A d S} c}_{E_{\mathrm{DM}}(X)}, \quad E_{\mathrm{DM}}(X)=r(X) m_{\text {AdS }}(X) c^{2},
$$


where the ratio $r(X):=E_{\mathrm{DM}}(X) / m_{\text {AdS }}(X) c^{2}$ should reflect to some extent the ratio dark matter/visible matter. $B y$, "to some extent", we mean that this individual ratio should be trivially larger that 1 , to be compared with the estimated global ratio $27 / 5=5.4$, and not too large in order to be consistent with most of the observed ratios halo mass:stellar mass. Typically, a galaxy with a stellar mass of about $2 \times 10^{8} M_{\odot}\left(M_{\odot}\right.$ is the solar mass) should have a dark matter mass of about $6 \times 10^{10} M_{\odot}$; see, for instance [3], with noticeable exceptions [6].

Assumption 2. The appearance of dark matter held over a period when the temperature(s) was (were) compatible with a phase of entities $X$, compatibility being understood in the sense of validity of the equipartition theorem applied to the quantum-oscillator-like energy spectrum of an AdS elementary system:

$$
k_{B} T_{X} \approx \hbar \varkappa_{A d S} \approx \approx \frac{2 r(X)}{3} m_{A d S}(X) c^{2} .
$$

The most probable spin $1 / 2$ candidates $X$ in agreement with the above assumptions are stable light quarks $u$ and $d$ when, at the "quark epoch" (about over $10^{-12} \mathrm{~s}$ till $10^{-6} \mathrm{~s}, \mathrm{~T}>10^{12} \mathrm{~K}$ ), the quark-gluon plasma experienced the phase transition, i.e., hadronization, which marked the beginning of the "hadron epoch" (about over $10^{-6}$ s till $1 s, T>10^{10} \mathrm{~K}$ ). The current estimate of the hadronization temperature for light quarks [28] is $T_{c f}=156.5 \pm 1.5 \mathrm{MeV} \approx 1.8 \times 10^{12} \mathrm{~K}$ ("chemical freeze-out temperature"). Thus, with $m_{\text {Ads }} \approx m$,

$$
T_{X} \approx 1.8 \times 10^{12} \mathrm{~K} \approx \frac{2 r(X)}{3} \frac{m(X) c^{2}}{k_{B}}
$$

Therefore, we obtain the estimates for quarks $u$ and $d$ :

$$
r(u) \approx 108, \quad r(d) \approx 49
$$

These values may be viewed as reasonable with regard to Assumption 1.

Moreover, the value of $T_{X}$ in Equation (5) yields AdS curvature $\varkappa_{A d S}$ and lifetime $\tau$ :

$$
k_{B} T_{X} \approx \hbar \varkappa_{A d S} C \equiv \frac{\hbar}{\tau} \Rightarrow \tau \approx 2.7 \times 10^{-23} \mathrm{~s}, \quad \varkappa_{A d S}^{-1} \approx 8 \mathrm{fm} .
$$

This AdS length scale $\varkappa_{\text {AdS }}^{-1} \approx 8 \mathrm{fm}$ is to be compared with the QGP typical distance scales, which exceed the size of the largest atomic nuclei (and the low typical momentum scale) (in the Pb case, $R_{\mathrm{Pb}} \approx 5.3788 \mathrm{fm}$ ).

Assumption 3. The pure AdS curvature energy decouples from the rest mass energy at the critical hadronization point and abides as a free component of the Universe along its posterior epochs.

\section{Discussion}

The conjectural interpretation we have proposed about the relation $E_{\mathrm{DM}}(X)=\frac{3}{2} \hbar \varkappa_{\mathrm{AdS}} c=\frac{3}{2} \hbar \omega_{\text {AdS }}$ as the remnant of the AdS zero-point energy of the spin 1/2 elementary system $X$ in the QGP period of the early Universe, which immediately follows the dS inflationary phase like an "AdS bounce" (AdS phase as anti inflation!) and which precedes the hadronization, requires of course a lot deeper analysis in terms of QCD, thermal QFT, and phase transition. Note that a QCD vacuum density due to conformal anomaly yields a Lorentz-invariant negative-valued contribution to the cosmological constant (see the review [7] and references therein). Unfortunately, this effect is negligible in comparison with the estimate in Equation (7). Moreover, the assumption of a AdS space-time should compel us to work within the framework of consistent thermodynamics, QFT, and QCD in AdS and not in Minkowski, a formidable program. Nevertheless, despite these drawbacks, one appealing aspect of our proposal is the fact that it rests upon the experimental evidence of the phase transition from hadronic confinement to QGP deconfinement, e.g., in CERN with SPS and LHC, or in BNL with RHIC and AGS (see [28] and references therein). Understanding the structure and phases of the QGP state of matter is certainly one of the main issues of modern nuclear physics. 
In this paper, we have advanced a guess about the emergence of dark matter based on some of the most basic symmetry considerations, by exploiting ideas coming from previous works like [21,25-27]. One may imagine that in the primordial QGP period happening just after inflation the massive constituents experience an effective geometric environment analogous to AdS during extremely short periods, and that the total zero-point energy resulting from those AdS phases subsists after hadronization. Like the cosmic microwave background (CMB) being a remnant from the recombination epoch of the universe $(\approx 379,000$ years, at $T \approx 3000 \mathrm{~K})$, when protons and electrons combined to form neutral hydrogen atoms, the dark matter would be a "relic" of the QGP epoch, totally free of any interaction but the gravitational one. It is indeed tempting to establish a parallel between dark matter and $\mathrm{CMB}$, since the latter is viewed as the emergence of the photon decoupling, precisely when photons started to travel freely through space rather than constantly being scattered by electrons and protons in plasma.

Finally, I cannot resist quoting Bacry and Lévy-Leblond in [10]:

...it is amusing to notice that, in the Newton Universe $N_{-}$, resulting from $c \rightarrow \infty$ contraction of AdS, the kinetic energy of the elementary system on the quantum level, that is,

$$
E_{\mathrm{kin}}^{N_{-}}:=\frac{I}{2}\left[P^{2}+\frac{1}{\tau^{2}} K^{2}\right], \quad I \equiv \text { Newtonian inertia }, \quad K \equiv \text { Newtonian boost generator }
$$

is quantized, which is not surprising in view of the "compactness" of the corresponding universe. The oscillator levels have a separation $\delta E_{\mathrm{kin}}^{N_{-}} \approx \hbar \tau^{-1}$ in agreement with the uncertainty principle, since $\tau$ may be thought of as the "lifetime" of this oscillating universe.

Funding: This research received no external funding.

Conflicts of Interest: The author declares no conflict of interest.

\section{References}

1. Planck Collaboration. Planck 2018 results XIII. Cosmological parameters. arXiv 2018, arXiv:1807.06209v1.

2. Baudis, L. The Search for Dark Matter. Eur. Rev. 2017, 26, 70-81. [CrossRef]

3. Behroozi, P.S.; Wechsler, R.H.; Conroy, C. The average star formation histories in dark matter halos from $z=0-8$. Astrophys. J. 2013, 770, 57. [CrossRef]

4. Van Dokkum, P.; Danieli, S.; Cohen, Y.; Merritt, A.; Romanowsky, A.J.; Abraham, R.; Brodie, J.; Conroy, C.; Lokhorst, D.; Mowla, L.; et al. A galaxy lacking dark matter. Nat. Lett. 2018, 555, 629-632. [CrossRef]

5. van Dokkum, P.; Danieli, S.; Cohen, Y.; Romanowsky, A.J.; Conroy, C. The Distance of the Dark Matter Deficient Galaxy NGC 1052DF2. Astrophys. J. Lett. 2018, 864, L18. [CrossRef]

6. van Dokkum, P.; Danieli, S.; Abraham, R.; Conroy, C.; Romanowsky, A.J. A Second Galaxy Missing Dark Matter in the NGC 1052 Group. Astrophys. J. Lett. 2019, 874, L5.

7. Pasechnik, R.; Šumbera, M. Phenomenological Review on Quark-Gluon Plasma: Concepts vs. Observations. Universe 2017, 3, 7. [CrossRef]

8. Newton, T.D.; Wigner, E.P. Localized States for Elementary Systems. Rev. Mod. Phys. 1949, 21, 400-406. [CrossRef]

9. Wigner, E.P. On Unitary Representations of the Inhomogeneous Lorentz Group. Ann. Math. 1939, 40, 149-204. [CrossRef]

10. Bacry, H.; Lévy-Leblond, J.-M. Possible Kinematics. J. Math. Phys. 1968, 9, 1605. [CrossRef]

11. Gürsey, F. Introduction to the de Sitter group. In Group Theoretical Concepts and Methods in Elementary Particle Physics; Gordon and Breach Science Publishers, Inc.: New York, NY, USA, 1964.

12. Fronsdal, C. Elementary particles in a curved space. Rev. Mod. Phys. 1965, 37, 221-224. [CrossRef]

13. Carroll, S.M.; Press, W.H.; Turner, E.L. The Cosmological Constant. Ann. Rev. Astron. Astrophys. 1992, 30, 499-542. [CrossRef]

14. Dixmier, J. Représentations intégrables du groupe de De Sitter. Bull. Soc. Math. Fr. 1961, 89, 9-41. [CrossRef]

15. Takahashi, R. Sur les représentations unitaires des groupes de Lorentz généralisés. Bull. Soc. Math. Fr. 1963, 91, 289-433. [CrossRef] 
16. Evans, N.T. Discrete series for the universal covering group of the $3+2$ de Sitter group. J. Math. Phys. 1967, 8, 170-184. [CrossRef]

17. Barut, A.O.; Böhm, A. Reduction of a class of $\mathrm{O}(4,2)$ representations with respect to $\mathrm{SO}(4,1)$ and $\mathrm{SO}(3,2)$. J. Math. Phys. 1970, 11, 2938-2945. [CrossRef]

18. Fronsdal, C. Elementary particles in a curved space. II. Phys. Rev. D 1974, 10, 589-598. [CrossRef]

19. Mizony, M. 3 semigroupes de causalité et formalisme hilbertien de la mécanique quantique. Publ. Dep. Math. Lyon 1984, 3B, 47-64.

20. Mickelsson, J.; Niederle, J. Contractions of representations of de Sitter groups. Commun. Math. Phys. 1972, 27, 167-180. [CrossRef]

21. Garidi, T.; Huguet, E.; Renaud, J. de Sitter waves and the zero curvature limit. Phys. Rev. D 2003, 67, 124028.

22. Bros J.; Gazeau, J.-P.; Moschella, U. Quantum Field Theory in the de Sitter Universe. Phys. Rev. Lett. 1994, 73, 1746. [CrossRef]

23. Garidi, T. What Is Mass in de Sitterian Physics? arXiv 2003, arXiv:hep-th/0309104.

24. Dooley, A.H.; Rice, J.W. On contractions of semisimple Lie groups. Trans. Am. Math. Soc. 1985, 289, $185-202$. [CrossRef]

25. Gazeau, J.-P.; Novello, M. The question of mass in (anti-) de Sitter spacetimes. J. Phys. A Math. Theor. 2008, 41, 304008. [CrossRef]

26. Gazeau, J.-P.; Novello, M. The Nature of $\Lambda$ and the Mass of the Graviton: A Critical View. Int. J. Mod. Phys. A 2011, 26, 3697-3720. [CrossRef]

27. Gazeau, J.-P.; Renaud, J. Relativistic harmonic oscillator and space curvature. Phys. Lett. A 1993, $179,67$. [CrossRef]

28. Andronic, A.; Braun-Munzinger, P.; Redlich, K.; Stachel, J. Decoding the phase structure of QCD via particle production at high energy. Nature 2018. [CrossRef]

(C) 2020 by the author. Licensee MDPI, Basel, Switzerland. This article is an open access article distributed under the terms and conditions of the Creative Commons Attribution (CC BY) license (http:/ / creativecommons.org/licenses/by/4.0/). 